\title{
The History of Development of Insulin Integration in Pharmacy Introduction Teaching
}

\author{
Zhi-ping WANG ${ }^{1,{ }^{*}}$, Fan YANG ${ }^{1}$ and Yi-fei WANG ${ }^{2}$ \\ ${ }^{1}$ College of Pharmacy, Guangdong Pharmaceutical University, 510006 China \\ ${ }^{2}$ Institute of Biological Medicine, Jinan University, 510632 China \\ ${ }^{*}$ Corresponding author: wzping_jshb@126.com
}

Keywords: Pharmacy introduction, Insulin, History of development, Teaching.

\begin{abstract}
Objective: In order to improve the learning efficiency, quality and the comprehensive ability of Pharmacy Introduction for nonpharmacy students in medical colleges and Universities.

Methods: In "Introduction", "Pharmacy", "Pharmaceutical analysis science", "Biopharmaceuticals" and "Pharmacology" teaching process, introduce the discovery of insulin and the Nobel Prize for Frederick G. Banting and John J.R. Macleod and Frederick Sanger and Dorothy Hodgkin and Rosalyn Sussman Yalow and George Richards Minot, the formulation and its preparation and clinical application of insulin, the pharmacopeia situation and quality control methods of insulin and its preparations, the 50th anniversary of the total synthesis of crystalline bovine insulin in China, the new pharmacological findings of insulin, respectively.

Results: The history of development of insulin integrates in Pharmacy Introduction teaching can cultivate achievement consciousness, motivate learning interests and exploration spirit and improve teaching efficiency and quality.

Conclusion: The thawing methods can improve the learning efficiency, quality and the comprehensive ability of Pharmacy Introduction for nonpharmacy students.
\end{abstract}

\section{Introduction}

Insulin is a peptide hormone produced by beta cells in the pancreas. It regulates the metabolism of carbohydrates and fats by promoting the absorption of glucose from the blood to skeletal muscles and fat tissue and by causing fat to be stored rather than used for energy. Insulin also inhibits the production of glucose by the liver. Except in the presence of the metabolic disorder diabetes mellitus and metabolic syndrome, insulin is provided within the body in a constant proportion to remove excess glucose from the blood, which otherwise would be toxic. When blood glucose levels fall below a certain level, the body begins to use stored glucose as an energy source through glycogenolysis, which breaks down the glycogen stored in the liver and muscles into glucose, which can then be utilized as an energy source. As a central metabolic control mechanism, its status is also used as a control signal to other body systems (such as amino acid uptake by body cells). In addition, it has several other anabolic effects throughout the body. When control of insulin levels fails, diabetes mellitus can result. As a consequence, insulin is used medically to treat some forms of diabetes mellitus. Patients with type 1 diabetes depend on external insulin (most commonly injected subcutaneously) for their survival because the hormone is no longer produced internally. Patients with type 2 diabetes are often insulin resistant and, because of such resistance, may suffer from a "relative" insulin deficiency. Some patients with type 2 diabetes may eventually require insulin if dietary modifications or 
other medications fail to control blood glucose levels adequately. Over $40 \%$ of those with Type 2 diabetes require insulin as part of their diabetes management plan[1].

Pharmacy Introduction is one of the important courses for students in non-pharmacy specialty in higher medicine colleges and universities, and is the basic class to help students to understand the pharmacy discipline and the pharmacy occupation. It is of great significance and necessity to open the course of Pharmacy Introduction. The curriculum take the medicine discovery, drugs production, quality control, drugs circulation and use, the medicine matter managements, as the master line. It introduces the corresponding pharmacy occupation content and the occupation development to the correlation knowledge and the skill request. It builds the concept of the occupation outline and the characteristic for the students from the pharmacy class specialty before the professional course study, and it is the guidance following curriculum study. The students feel the curriculum covering with widespread abstract, monotonous in the study process. The history of development of insulin integrates in Pharmacy Introduction teaching introduces the knowledge below in "Introduction", "Pharmacy", "Pharmaceutical analysis science", "Biopharmaceuticals" and "Pharmacology" teaching process respectively: the discovery of insulin and the Nobel Prize for Frederick G. Banting and John J.R. Macleod and Frederick Sanger and Dorothy Hodgkin and Rosalyn Sussman Yalow and George Richards Minot, the formulation and its preparation and clinical application of insulin, the pharmacopeia situation and quality control methods of insulin and its preparations, the 50th anniversary of the total synthesis of crystalline bovine insulin in China, the new pharmacological findings of insulin. Through the vivid scene reappearance, it arouses students' national sense of pride and achievement consciousness fully, and then stimulates the student enormous study interest and science exploration spirit, enhances the teaching effect and the quality of teaching effectively.

\section{Methods}

\section{"Introduction" Integrates the Discovery of Insulin and the Nobel Prize}

The Nobel Prize committee in 1923 credited the practical extraction of insulin to a team at the University of Toronto and awarded the Nobel Prize to two men: Frederick G. Banting (1891-1941) and J.J.R. Macleod (1876-1935). They were awarded the Nobel Prize in Physiology or Medicine in 1923 for the discovery of insulin. Banting, insulted that Best was not mentioned, shared his prize with him, and Macleod immediately shared his with James Collip. Despite the Nobel decision and the well-publicized splitting of the cash prizes, Banting and Best are often credited in the international arena with the discovery of insulin and its transformation into a life-sustaining therapy for diabetes. Everyone warmed to the Hollywood-like myth of two novices, a surgeon and a medical student, who came out of nowhere and during their first summer, while the professor who provided the lab was away on "vacation", succeeded in a task that had stymied many professional researchers who came before them. The patent for insulin was sold to the University of Toronto for one half-dollar $[2,3]$.

The primary structure of insulin was determined by British molecular biologist Frederick Sanger (1918-2013). It was the first protein to have its sequence be determined. He was awarded the 1958 Nobel Prize in Chemistry for this work. He won two Nobel Prizes for chemistry, but we claim him for molecular biology because the methods he developed for sequencing proteins and nucleic acids provide the basis for much of what we do today[4,5]. In 1969, after decades of work, Dorothy Hodgkin 
(1910-1994) determined the spatial conformation of the molecule, the so-called tertiary structure, by means of X-ray diffraction studies. She had been awarded a Nobel Prize in Chemistry in 1964 for the development of crystallography [6]. Rosalyn Sussman Yalow (1921-2011) received the 1977 Nobel Prize in Medicine for the development of the radioimmunoassay for insulin [7]. George Richards Minot (1885-1950), co-recipient of the 1934 Nobel Prize for the development of the first effective treatment for pernicious anemia, had diabetes mellitus. Dr. William Castle observed that the 1921 discovery of insulin, arriving in time to keep Minot alive, was therefore also responsible for the discovery of a cure for pernicious anemia [8].

\section{"Pharmacy" Integrates Insulin Formulation and Clinical Application}

The formulations of insulin are insulin injection, isophane insulin injection, insulin zinc injection, amorphous insulin zinc injection, crystalline insulin zinc injection, insulin zinc protamine injection, insulin human injection, insulin glargine injection, biphasic insulin injection, biphasic isophane insulin injection, insulin lispro injection, protamine zinc insulin injection, human insulin isophane suspension and human insulin injection, isophane insulin human suspension, extended insulin zinc suspension, prompt insulin zinc suspension, insulin human zinc suspension, extended insulin human zinc suspension, mainly. Insulin is usually taken as subcutaneous injections by single- use syringes with needles, via an insulin pump, or by repeated-use insulin pens with disposable needles. Inhaled insulin is also available in U.S. market now. Unlike many medicines, insulin currently cannot be taken orally because, like nearly all other proteins introduced into the gastrointestinal tract, it is reduced to fragments (even single amino acid components), whereupon all activity is lost. There has been some research into ways to protect insulin from the digestive tract, so that it can be administered orally or sublingually. While experimental, several companies now have various formulations in human clinical trials.

\section{"Pharmaceutical analysis science" Integrates the Pharmacopeia Situation and Quality Control}

Insulin is a very old protein that may have originated more than a billion years ago. The molecular origins of insulin go at least as far back as the simplest unicellular eukaryotes. Apart from animals, insulin-like proteins are also known to exist in Fungi and Protista kingdoms. The human insulin protein is composed of 51 amino acids, and has a molecular mass of $5808 \mathrm{Da}$. It is a dimer of an A-chain and a B-chain, which are linked together by disulfide bonds. Insulin's structure varies slightly between species of animals. Insulin from animal sources differs somewhat in "strength" (in carbohydrate metabolism control effects) from that in humans because of those variations. Porcine insulin is especially close to the human version.

\section{"Biopharmaceuticals" Integrates the Total Synthesis of Insulin}

Purified animal-sourced insulin was the only type of insulin available to diabetics until genetic advances occurred later with medical research. The amino acid structure of insulin was characterized in the early 1950s by Frederick Sanger, and the first synthetic insulin was produced simultaneously in the labs in the early 1960s in China. The first genetically engineered, synthetic "human" insulin was produced using E. coli in 1978 by Arthur Riggs and Keiichi Itakura at the Beckman Research Institute of the City of Hope in collaboration with Herbert Boyer at Genentech. Genentech, founded by Swanson, Boyer and Eli Lilly and Company, went on in 1982 to sell the first commercially available biosynthetic human insulin under the brand name 
Humulin. The vast majority of insulin currently used worldwide is now biosynthetic recombinant "human" insulin or its analogues.

Recombinant insulin is produced either in yeast (usually Saccharomyces cerevisiae) or E. coli. In yeast, insulin may be engineered as a single-chain protein with a KexII endoprotease (a yeast homolog of PCI/PCII) site that separates the insulin A chain from a c-terminally truncated insulin B chain. A chemically synthesized c-terminal tail is then grafted onto insulin by reverse proteolysis using the inexpensive protease trypsin; typically the lysine on the c-terminal tail is protected with a chemical protecting group to prevent proteolysis. The ease of modular synthesis and the relative safety of modifications in that region accounts for common insulin analogs with c-terminal modifications (e.g. lispro, aspart, glulisine). The Genentech synthesis and completely chemical synthesis such as that by Bruce Merrifield are not preferred because the efficiency of recombining the two insulin chains is low, primarily due to competition with the precipitation of insulin B chain.

\section{"Pharmacology" Integrates the New Pharmacological Findings of Ins ulin}

Biosynthetic human insulin (insulin human rDNA, INN) for clinical use is manufactured by recombinant DNA technology. Biosynthetic human insulin has increased purity when compared with extractive animal insulin, enhanced purity reducing antibody formation. Researchers have succeeded in introducing the gene for human insulin into plants as another method of producing insulin ("biopharming") in safflower. This technique is anticipated to reduce production costs.

Several analogs of human insulin are available. These insulin analogs are closely related to the human insulin structure, and were developed for specific aspects of glycemic control in terms of fast action (prandial insulins) and long action (basal insulins). The first biosynthetic insulin analog was developed for clinical use at mealtime (prandial insulin), Humalog (insulin lispro), it is more rapidly absorbed after subcutaneous injection than regular insulin, with an effect 15 minutes after injection. Other rapid-acting analogues are NovoRapid and Apidra, with similar profiles. All are rapidly absorbed due to sequence that will reduce formation of dimers and hexamers (monomeric insulins are more rapidly absorbed). Fast acting insulins do not require the injection-to-meal interval previously recommended for human insulin and animal insulins. The other type is long acting insulin; the first of these was Lantus (insulin glargine). These have a steady effect for an extended period from 18 to 24 hours. Likewise, another protracted insulin analogue (Levemir) is based on a fatty acid acylation approach. A myristyric acid molecule is attached to this analogue, which in turn associates the insulin molecule to the abundant serum albumin, which in turn extends the effect and reduces the risk of hypoglycemia. Both protracted analogues need to be taken only once daily, and are used for type 1 diabetics as the basal insulin. A combination of a rapid acting and protracted insulin is also available, making it more likely for patients to achieve an insulin profile that mimics that of the body's own insulin release [9-11].

\section{Conclusion}

The history of development of insulin integrates in Pharmacy Introduction teaching can cultivate achievement consciousness, motivate learning interests and exploration spirit and improve teaching efficiency and quality. The thawing methods can improve the learning efficiency, quality and the comprehensive ability of Pharmacy Introduction for nonpharmacy students. 


\section{References}

[1] P. Sonksen, J. Sonksen, Insulin: understanding its action in health and disease, Br. J. Anaesth. 85 (2000) 69-79.

[2] T.N.K. Raju, A mysterious something: The discovery of insulin and the 1923 Nobel Prize for Frederick G. Banting (1891-1941) and John J.R. Macleod (1876-1935), Acta Paediatrica. 95 (2006) 1155-1156.

[3] J. Roth, S. Qureshi, I Whitford, et al., Insulin's discovery: New insights on its ninetieth birthday, Diabetes Metab. Res. Rev. 28 (2012) 293-304.

[4] S. Brenner, Retrospective: Frederick Sanger (1918-2013), Science. 343 (2014) 262.

[5] J. Walker, Frederick Sanger (1918-2013), Nature. 505 (2014) 27.

[6] M. Perutz, Obituary: Dorothy Hodgkin (1910-94), Nature. 371 (1994) 20.

[7] G. Watts, Obituary: Rosalyn Sussman Yalow, Lancet. 378 (2011) 122.

[8] W.B. CASTLE, The contributions of George Richards Minot to experimental medicine, New. Engl. J. Med. 247 (1952) 585-592.

[9] F. Koumanov, V.J. Pereira, J.D. Richardson, et al., Insulin regulates Rab3-Noc2 complex dissociation to promote GLUT4 translocation in rat adipocytes, Diabetologia. 58 (2015) 1877-1886.

[10] R.I. Meijer, E.H. Serné, H. Ibrahim Korkmaz, et al., Insulin-induced changes in skeletal muscle microvascular perfusion are dependent upon perivascular adipose tissue in women, Diabetologia. 58 (2015) 1907-1915.

[11] C. Lamalle, D. Roland, J. Crommen, et al., Separation of human, bovine, and porcine insulins, three very closely related proteins, by micellar electrokinetic chromatography, Electrophoresis. 36 (2015) 2504-2506. 